\title{
Buchbesprechungen / Recensions critiques / Book Reviews
}

\author{
Die Auswahl der Bücher zur Rezension behalten sich die Redaktion und die beiden für diese Rubrik \\ Verantwortlichen vor. Unverlangt eingesandte Buchbesprechungen werden nicht veröffentlicht.
}

Le choix des livres qui font l'objet d'une recension critique est effectué par la rédaction et par les deux responsables de cette rubrique. Les recensions non sollicitées ne sont pas publiées.

Books to be reviewed are selected by the Editor and the two Book Review Editors. Unsolicited reviews are not published.

Fellay-Favre, Elisa: Le travail social et le recours à la fiction. La relation entre assistante social.e et bénéficiaire de l'aide sociale financière en Suisse. Paris, L'Harmat$\tan , 2019,170 \mathrm{p}$.

Quelle relation se noue entre assistants sociaux et bénéficiaires de l'aide sociale financière en Suisse, et comment en rendre compte? Cet ouvrage s'intéresse à ce qui est en jeu, du point de vue des professionnels impliqués, dans la relation d'aide qu'ils tentent d'instaurer avec les bénéficiaires et propose la métaphore théâtrale comme clé de lecture de ce qui s'y passe. L'auteure cherche ainsi à éclairer une dimension selon elle importante de la relation d'aide, à savoir qu'elle serait une expérience fictionnelle. Il s'agit dès lors de montrer, comme l'explique Marc-Henry Soulet dans la préface, "comment la fiction peut s'avérer être un puissant levier de mobilisation des personnes en quête de ressaisissement en développant une compétence «imaginante q qui favorise le passage d'une situation réelle posant problème à une immersion fictionnelle (...)»(p. 11), dans laquelle ces personnes expérimentent certains remaniements de l'image de soi et de leurs manières d'agir.

Dans la première partie de l'ouvrage, l'auteure expose le cheminement intellectuel qui la conduit à prendre au sérieux la place occupée par la fiction dans les pratiques du travail social. L'idée selon laquelle l'activité développée par ces professionnels ne consiste ni plus, ni moins, qu'à conduire l'usager à réaliser une expérience fictionnelle s'est forgée au cours d'une enquête empirique. L'auteure a mené onze entretiens auprès d'assistants sociaux et des observations ont été conduites en immersion dans les bureaux de l'aide financière d'un canton suisse (assez peu de détails sur la démarche méthodologique sont livrés au lecteur). Ces travailleurs sociaux sont chargés de la réinsertion de personnes incitées à participer à des mesures d'insertion sociale ou professionnelle, en contrepartie d'une aide financière octroyée par le service cantonal d'action sociale et la commune où ils résident. L'auteure montre que ces professionnels, disposant de très peu de ressources pour transformer la situation des bénéficiaires, n'ont cependant pas renoncé à susciter des changements dans l'existence de ces derniers. À travers l'aide relationnelle, ils cherchent à modifier la représentation que le bénéficiaire a de lui-même et l'estime qu'il se porte, dans la perspective qu'il accomplisse lui-même des actes lui permettant de sortir de l'impasse dans laquelle il se trouve. Tout l'objet du livre est précisément de documenter la manière dont ces travailleurs sociaux élaborent ce soutien relationnel et régulent leur engagement dans la relation afin que celle-ci produise l'effet escompté. Ces professionnels s'attachent pour l'essentiel à créer un état d' "immersion fictionnelle" visant à susciter de la part du bénéficiaire, des émotions, des sentiments, des réflexions, qui le mèneront sur la voie d'un changement positif 
à l'égard de lui-même. Dans le but d'étayer cette thèse originale, l'auteure montre que si le rôle de la fiction dans la relation en travail social constitue une dimension rarement relevée, elle est malgré tout présente dans les analyses de certains sociologues spécialistes de ce domaine, tels que D. Vrancken, ou P. Vidal-Naquet. Pour apporter sa pierre à l'édifice, l'auteure s'inspire de travaux en philosophie de l'esthétique et des arts, tels ceux de Jean-Marie Schaeffer, ce dernier ayant particulièrement théorisé cette forme d'état mental particulier qui correspond à l' «immersion fictionnelle». Il définit l'immersion fictionnelle en cinq critères dont l'auteure affirme qu'ils correspondent en tous points au fonctionnement de la relation d'aide: « un état d'imagination active», "un dédoublement des mondes", "un état dynamique», "un état d'investissement affectif», "un rapport entre illusion et croyance».

La première partie de l'ouvrage regorge de propositions théoriques stimulantes, construites sur la base des résultats d'enquête, qui permettent d'éclairer la manière dont ces professionnels construisent leur posture et leur rôle. Premièrement, la notion de "procédure de suspension consentie de l'incrédulité» (p. 48) rend intelligible une action volontairement engagée par les professionnels, qui consiste à ne pas mettre en doute ni porter un regard critique sur le récit et l'attitude du bénéficiaire. Deuxièmement, en s'inspirant de concepts existants - ceux d'agir faible (M.-H. Soulet), de prudence (Artistote, F. Champy), de ruse (P. VidalNaquet) - l'auteure forge la notion d' "agir discret» (p. 67), qui permet de dépasser une vision manichéenne des pratiques, tantôt décrites comme exclusivement basées sur un rapport de confiance, tantôt principalement orientées par une visée de contrôle. L'agir discret permet de concevoir la manière dont les professionnels s'arrangent pour rendre ténue la surveillance qu'ils exercent sur les usagers dans le but de préserver la relation.

Dans la seconde partie, l'auteure analyse le fonctionnement de la relation à travers une étude de cas, considérée comme exemplaire des relations qui se nouent entre bénéficiaires et professionnels. L'analyse se fonde sur le récit, par un assistant social, de la façon dont il s'est trouvé impliqué dans la relation avec un bénéficiaire. Le premier chapitre met l'accent sur la manière dont ces professionnels s'attachent à créer des ambiances particulières et des rituels visant un rapprochement entre les parties et l'instauration d'un univers familier. Le second chapitre met au jour l'implication émotionnelle des professionnels. L'analyse révèle combien la relation nouée dans ces espaces institutionnels ne se construit pas selon des procédures standardisées, des guides de bonnes pratiques et des objectifs établis. Les professionnels cherchent au contraire à se montrer sensibles au vécu de l'usager et à ses ressentis. Ils tentent de cerner ce qui fait réellement sens pour lui, afin d'identifier les ressources susceptibles d'être mobilisées dans une perspective de changement. Le troisième chapitre décrit les différentes étapes d'une relation censée s'établir sur un rapport de confiance. Certes, les épreuves mettant à mal le lien de confiance sont nombreuses. Celui-ci constituerait cependant leur outil principal pour nourrir l'estime de soi des usagers, considérée comme un levier essentiel de transformation de leur vie. En accordant du crédit aux capacités d'autonomie des usagers, ces professionnels les "convoquent en sujets capables et dignes de confiance, d'attention et de futur" (p. 142) et, ce faisant, leur offrent un espace d'opportunités pour se penser comme tels.

Selon nous, la mise en exergue d'une dimension fictionnelle de la relation d'aide est une piste crédible pour rendre compte de ce qui se passe sur les scènes actuelles du travail social. Pendant longtemps, les "fictions nécessaires à l'accomplissement d'un travail sur autrui» (Dubet, 2002, 48) - permettant d'en "réduire les dimensions tragiques» notamment (Ibid., 47) - étaient produites en amont des pratiques, dans le cadre de ce que François Dubet nomme «le programme institutionnel». Mais qu'en est-il de la fiction quand ce programme institutionnel n'opère plus ? Sous la plume d'Elisa Fellay-Favre, la fiction 
réapparait comme une nécessité de moyens pour les acteurs, au regard de ce qu'elle rend possible. Elle constitue l'ultime ressource d'un travailleur social désormais "pieds et poings liés» (p. 57). Quand les consignes officielles réduisent le rôle professionnel à une fonction de contrôle des bénéficiaires, quand le temps vient à manquer (logique d'efficacité) ou quand les savoirs professionnels sont mis en défaut (contexte d'incertitude), alors, oui, il ne reste que la fiction pour (re) mettre le travailleur social en capacité d'agir. Néanmoins, dans la perspective d'asseoir et de consolider cette thèse, des liens avec certaines ressources théoriques nous paraissent devoir être réaffirmés, justifiant aussi certains prolongements méthodologiques.

Primo, contrairement à ce que d'aucuns pourraient penser, l'activité fictionnelle n'est ni une pratique extravagante inventée par des professionnels farfelus, ni l'effet produit par l'usage de techniques d'ordre psychologique. Au contraire, l'activité fictionnelle est somme toute une activité sociale tout à fait ordinaire et quotidienne car notre perception de la réalité relève ... : d'une fiction, Goffman (1974) l'a bien montré! Comme le souligne Ogien sur cette question de la frontière floue entre réalité et fiction, des "fictions opératoires" (Ogien, 2014, 14) à l'origine des «cadres primaires» orientent en effet nos perceptions, fixent «la représentation de la réalité, donnent à l'individu l'impression que la réalité est bien ce qu'elle est" (Nizet et Rigaux, 2005, 72). Qui plus est, le concept de "modalisation" permet à Goffman de montrer que des fictions sont parfois délibérément produites. Lactivité fictionnelle constitue donc un objet de recherche sociologique de plein droit tant celle-ci est au cœur de la vie sociale.

Secundo, pour parvenir à une compréhension complète du type de relation qui se noue dans le cadre de l'activité fictionnelle, il nous semblerait utile que l'analyse prenne davantage en compte, par-delà les constructions narratives des professionnels, l'étude des situations et des interactions effectives. Comment s'ancre l'activité fictionnelle au travers de situations qui, elles, sont bien réelles? Qu'est-ce qui se joue pour l'usager sur ces scènes et comment y prend-il part? Le caractère situé de l'activité fictionnelle pourrait de ce point de vue être davantage mis en relief à travers la notion d' "ordre pactisé» (Giuliani, 2013, 127) à laquelle l'auteure se réfère longuement. L'usage de cette notion implique de décrire comment professionnels et usagers, pris dans des réalités multiples, construisent un accord (des pactes) dans ces situations où, précisément, il n'existe pas d'accord minimal ni sur ce qui fait problème ni sur la manière de se coordonner en vue de le résoudre. Menée à son terme, cette perspective permettrait de mieux mettre en exergue la dimension pratique de l'activité fictionnelle, et de contrer l'idée que celle-ci relèverait uniquement de la subjectivité des professionnels, pire, d'une illusion biographique entretenue par la rhétorique professionnelle. En conclusion, la connaissance des tenants et aboutissants de cette immersion fictionnelle judicieusement engagée par Elisa Fellay-Favre dans ce livre est appelée à se prolonger à travers l'étude des situations d'interactions concrètes qui se développent dans ces contextes institutionnels dont les usagers sont partie prenante.

\section{Dubet, Francois. 2002. Le déclin de l'institution.} Paris: Seuil.

Giuliani, Frédérique. 2013. Accompagner. Le travail social face à la précarité durable. Rennes: Presses Universitaires de Rennes.

Goffman, Erving. 1974. Les cadres de l'expérience. Paris: Les éditions de Minuit.

Nizet, Jean et Nathalie Rigaux. 2005. La sociologie de Erving Goffman. Paris: Editions La Découverte.

Ogien, Albert. 2014. Expression, communication, conceptualisation. Un itinéraire dans le travail de Goffman. Occasional Paper 17. Institut Marcel Mauss http://cems.ehess.fr/ docannexe/file/3163/op_17_goffman_ogien. pdf (01.03.2021).

Frederique.giuliani@unige.ch 